\title{
Point d'étape en fin 2006 sur l'assurance de la sécheresse géotechnique en France
}

\section{R. NUSSBAUM}

Directeur de la Mission risques naturels (MRN) roland.nussbaum@mrn.

gpsa.f
Depuis 1989, une grande partie tes dommages causés par la sécheresse gếotechnique a été prise en charge, au titre du régime français d'assurance des catastrophes naturelles « Catnat $\rightsquigarrow$. Trois pics de sinistralité ont étê observés, en 1990, puis en 1996-98, avec un épisode paroxystique attribué à la canicule de 2003 , à l'origine de sérieuses préoccupations sur l'équilibre du régime et son évolution, de la part des pouvoirs publics, comme des assureurs. La réforme projetée n'ayant pas été présentée au Parlement, les assureurs s'en tiennent pour l'heure à exprimer des orientations sur le volet sécheresse de ce projet, tant sur le plan de la prévention que des conditions d'assurance.

Mots-clés : sécheresse géotechnique, sinistralitê, êquilibre économique, régime Catnat, projet de réforme, assureurs, prévention.
Mission des sociétés d'assurance pour la connaissance et la prêventoon des riscues naturels, associaton entre la Fédération française des sociétés d'assurances [FFSA] et le Groupement des entreprises muthelle d'assurances [GEMA]

\section{Insurance conditions of subsidence due to drought period at the end of the year 2006 in France}

\begin{abstract}
0
0
$\frac{6}{6}$
6
Since 1989, most of subsidence claims have been taken over within the French "CatNat" insurance regime. Three peaks of loss accumulations have been observed, in 1990 , the between 1996 and 1998, with an extraordimary one attributed to the 2003 heat wave, which caused serious preoccupations on the equilibrium of the regime and its evolution, from the side of Public authorities as well as from the insurers'side. As the projected reform has not yet been presented to Parliament, the insurers stick to present their views on the part of this project regarding subsicience coverage, about prevention and on the insurance conditions.
\end{abstract}

Key words: Subsidence, loss records, economic equilibrim, Cat Nat insurance regime, project of reform, insurers, prevention. 


\section{1}

\section{Introduction}

Un nouveau pic de sinistralité attribuable à des formes de sécheresse géotechnique, sưte à la canicule de 2003, est à l'origine de nouvelles préoccupations sur l'équilibre économique du régime d'inctemnisation des catastrophes naturelles ${ }^{1}$. Une procédure exceptionnelle a été lancée par le Gouvemement pour accorder une aide de 218 millions d'euros aux propriétaires de maisons les plus gravement endommagées dans les communes n'ayant pas bénéficiế d'un arrêté CatNat (3 400 sur 7000 qui en ont fait la demande). Mais ces moyens ont été jugés largement insuffisants, de sorte que les « oubliés n de la sécheresse 2003 réclament 1,4 milliard d'euros?.

Le diagnostic et des recommandations de réforme du régime CatNat ont fait l'objet d'un rapport d'inspection générale interministérielle de décembre 2005, avec un rapport particulier consacré à la sécheresse

\footnotetext{
t Une sikuation analogue 5 était dejă produite ern 2000, swite aux deux

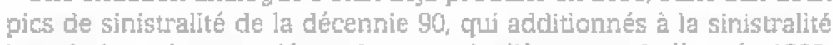
inondations de cette décennỉe el particulièrementi de l'année 1999. avait déjă conduit l'Etat â prendre des mesures drastiques pour réta blir l'équiłbre économique du régime (mise en jeu de la garantie de I"Etat sur la CCR, relevement du taux de la surprine de 9 à $12 \%$ \%),

2 Les Échos, 15 mars 2007.

3nspection générale des finarces, Conseil génêral des ponts et chausşées, Inspection générale de l'enviromenent. lispection générale de l'administration : Mission d'enquête sur Je régirne d'indemuisation des victues des catastrophes naturelles, septembre 2006.
}

Néanmoins, la réforme projetée suite à ce rapport n'ayant pas encore eu lieu et dans le contexte tendu et non réglé rappelé ci-dessus pour 2003, on se limitera ici :

- à un bref commentaire de l'évolution de la sinistralitế et de son impact sur l'équilibre économique du régime, pour mémoire :

- aux quelques éléments de position pris d'un commun accord par les assureurs sur les orientations de réforme à ce jour, dans ce domaine spécifique.

\section{2}

\section{Évolution de la sinistralité}

Le graphique ci-dessous reproduit les coûts annuels de la sécheresse géotechnique, estimés respectivement par la CCP et la FFSA, depuis sa prise en compte dans le régime, en 1989, jusqu'à l'année 2002, qui a précédé le pic de 2003 (source : rapport citê).

Le coût total pour la période est de l'ordre de 3 milliards d'euros, avec un coût moyen par sinistre de $10,5 \mathrm{k} €$ et par conmune sinistrée de 500 à $600 \mathrm{k} \in$.

L'évolution de la sinistralité en trois cycles et les niveaux de coûts moyens sont tout à fait similaires au Royaume-Unt.

Les périodes de sinistralité élevée se traduisent directement en décroissance de la provision d'égalisation de la Caisse centrale de réassurance (CCP), sorte de baromètre de l'équilibre économique du régime CatNat (cf. graphique ci-dessous, source CCR).

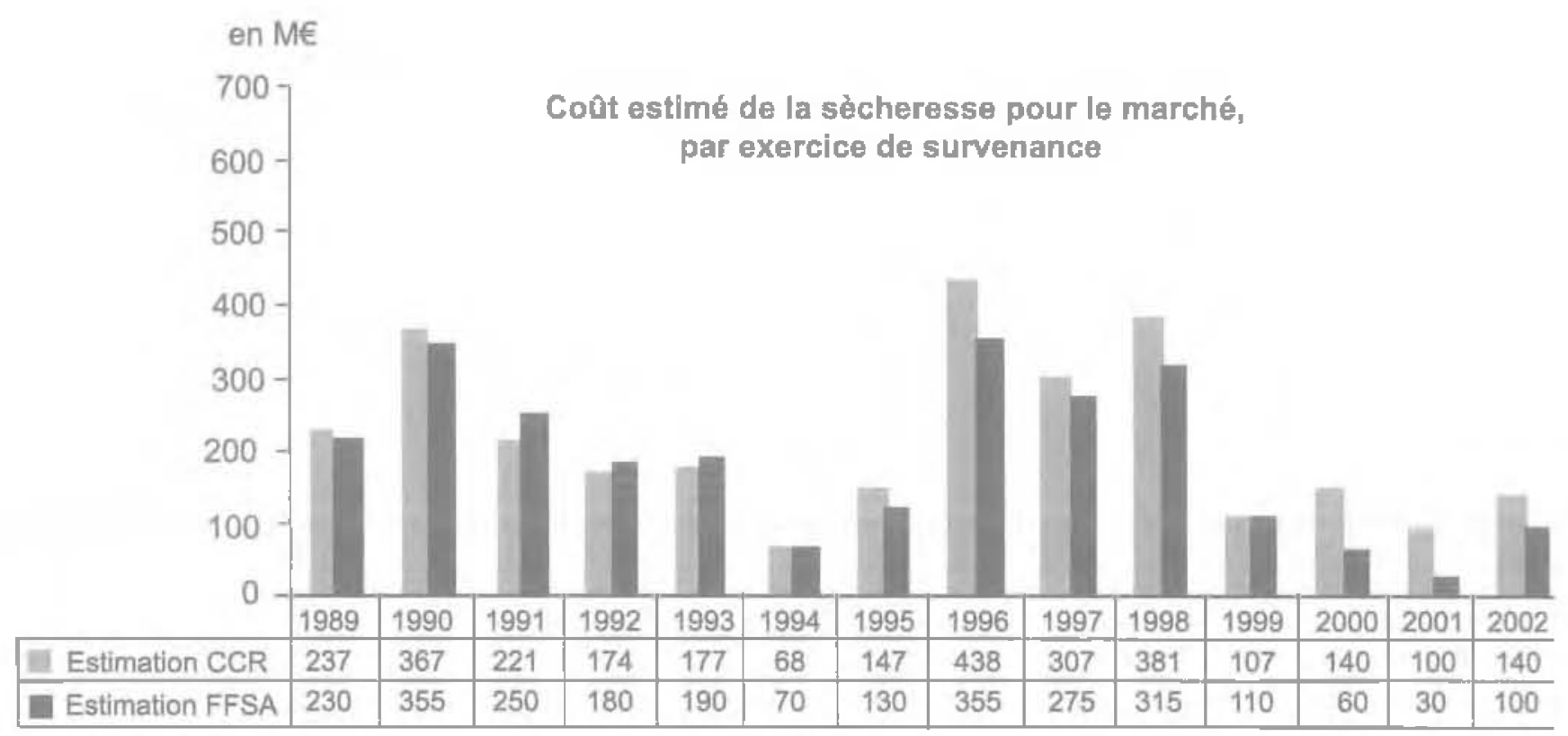


Évolution de la provision d'égalisation et du chiffre d'affalres de la CCR

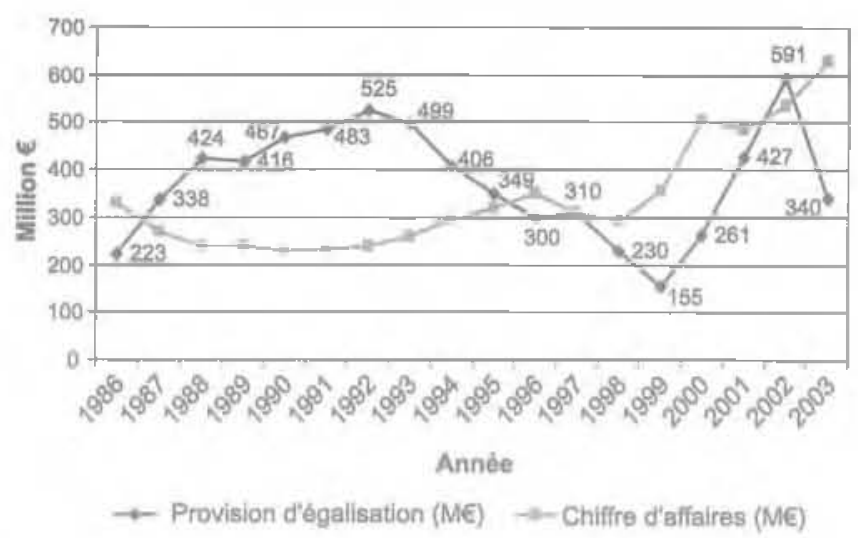

me 2

\section{Les assureurs et le projet de réforme}

Sur la prévention, les assureurs FFSA et GEMA ont retenu comme particulièrement importants, parmi les orientations proposées par la mission d'inspection générale, les points suivants:

- l'élaboration d'un zonage national et l'obligation réglementaire d'information de l'acquéreur d'un terrain ${ }^{4}$;

- une définition caractérisant les dommages couverts en sécheresse comme ceux qui portent atteinte à la solidité de la structure du bâtiment:

"Cette disposition a pris effet en juin 2006, en application de ta toi Bachelot, seulement dans le cas oủ un PPR a été prescrit ou approusvé.

- la mise en place de mesures de prévention accrues par les pouvoirs publics contre le risque sêcheresse et notamment la fixation de règles de construction tenant compte de la nature des sous-sols.

Sur le seul volet sécheresse du projet de réforme de la loi d’indernnisation quí leur a été présenté à ce jour, les assureurs se sont également déclarés favorables aux dispositions suivantes:

- cue le bénéfice de la garantie ne soit plus accordé, lorsque les dommages apparaissent plus de deux ans après le début du phénomène:

- que les ouvrages ne bénéficient plus de la garantie, lorsque les dommages surviennent au cours du délai de prescription décennal, à compter de la date de réception des travaux de construction du bâtiment ,

- que l'indemnisation ne couvre que les réparations nécessaires au confortement de là solidité de la structure de l'ouvrage.

Mais il importe que ces dispositions ne s'appliquent qu'aux bâtiments dont la construction a été entreprise postérieurement à la prise d'effet de la loi et des décrets d'application, puisqu'elles sont corrélées à la mise en place des mesures de prévention renforcées sous forme de règles impératives de construction, tenant compte de la nature des sous-sols.

\section{Conclusion}

Si de telies orientations devraient permettre de maintenir la sécheresse géotechnique dans le champ du régime CatNat, avec de meilleures garanties de pérennité, il est également important qu'une issue équitable soit trouvée par les parties concernées, pour tous les cas de sinistres avérés, datant de la canicule 2003 , et qui seraient encore en suspens. 